\title{
Indirect determination of soil water content
}

\author{
Philippe Cosenza ${ }^{1, a}$ \\ ${ }^{1}$ University of Poitiers, CNRS, UMR 7285 IC2MP, ENSI Poitiers, 86073 Poitiers cedex 09, France
}

\begin{abstract}
This manuscript reviews the recent developments in indirect methods used to measure soil watent content (SWC). It focuses on three types of methodological approaches: (i) high-frequency electromagnetic methodologies (dielectric methods and Ground Penetrating Radar), (ii) low-frequency electromagnetic methods (resistivity method and spectral induced polarization-SIP) and (iii) emerging methods (laboratory and ground-based nuclear magnetic resonance-NMR techniques and methods based on fiber optic temperature sensing systems). For each method the physical principles and the corresponding methodology are briefly described first, followed by the recent methodological advances. These recent advances address the following issues: (a) integration of different types of sensors to design new multi-functional devices, (b) miniaturization of low-cost and easily-installed electromagnetic sensors, (c) fusion and assimilation of data acquired from multiple modalities with different sampling rates and spatial resolutions, (d) physical understanding of spectra and distributed parameters measured by SIP and NMR methods, (e) improvement of geophysical inversion techniques combined with fast data acquisitions (4D monitoring of SWC).
\end{abstract}

\section{Introduction}

Soil water content (SWC) is a key parameter to understand the mechanical behavior of unsaturated soils. It controls many mechanical characteristics (e.g., plasticity, strength, desiccation cracking) and plays an important role in the stability of numerous geotechnical systems (engineered earth structures, landslides and artificial slopes such as embankments and cuttings). In recent decades, a variety of innovative methodologies used to measure SWC at different scales has been developed. These methodologies include mainly electrical and electromagnetic probes and geophysical techniques (i.e., non-invasive and non-destructive methods with a rapid spatial coverage and high-resolution of measurements).

This contribution reviews the recent developments in the indirect and geophysical methods used to measure SWC. Contrary to the standard gravimetric method, these approaches are indirect because they measure a physical parameter which is sensitive to the amount of soil water: they relate this physical parameter (or its variation) to SWC (mainly volumetric SWC in the following). In addition, this review focuses on laboratory and "local" field scales (typically $0.1-100 \mathrm{~m}$ ) relevant in geotechnical engineering; the remote sensing techniques operating on regional scales are not considered.

Three groups of methodological approaches used to estimate SWC are presented and discussed: (i) highfrequency electromagnetic methodologies often referred to dielectric methods, (ii) low-frequency electromagnetic methods (resistivity method and spectral induced

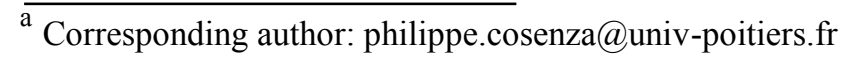

polarization) and finally (iii) emerging methods (nuclear magnetic resonance- NMR techniques and methods based on fiber optic temperature sensing systems). For each method the physical principles and the corresponding methodology are briefly described first, followed by the recent methodological advances obtained in the last years.

\section{High-frequency electromagnetic methods}

\subsection{Physical principles and methodologies}

High-frequency (HF) electromagnetic methods are based on the measurement of the soil relative dielectric permittivity $\varepsilon$ which is a complex parameter given by:

$$
\varepsilon(\omega)=\varepsilon^{\prime}(\omega)-j\left[\varepsilon^{\prime \prime}(\omega)+\frac{\sigma_{D C}}{\omega \varepsilon_{0}}\right]
$$

where $\varepsilon^{\prime}$ and $\varepsilon^{\prime \prime}$ are the real and imaginary component, respectively (both adimensionless variables); $\omega$ is the angular frequency of the imposed electromagnetic field ( $\omega=2 \pi f$ with frequency, $f$ expressed in Hertz); $j$ is the imaginary unit $\left(j^{2}=-1\right) ; \varepsilon_{0}\left(8.85410^{-12} \mathrm{~N}^{-1} \cdot \mathrm{m}^{-2} \cdot \mathrm{C}^{2}\right)$ is the vacuum dielectric permittivity and $\sigma_{D C}$ is the direct current (DC) electrical conductivity (S. $\left.\mathrm{m}^{-1}\right)$.

HF electromagnetic methods take advantage of the differences in real relative permittivity values, $\varepsilon^{\prime}$, between soil phases; the water, air and solid phases exhibit real relative permittivity values of approximately 80,1 and 3-10, respectively. 
HF electromagnetic sensors can be schematically classified in two groups: (a) transmission line methods such as time-domain reflectometry (TDR) and time domain transmission (TDT) and (b) capacitances probes often termed HF impedance measurements.

In the first group, TDR and TDT methods both use the propagation velocity of an electromagnetic wave along a pair (or a parallel group) of metallic rods to obtain the permittivity of the geomaterial in which they are embedded. TDR probes are characterized by the rise time of the step pulse used to generate the electromagnetic propagation; the shorter the time, the higher the frequency content of the signal. Generally, rise times shorter than 300 ps are used (e.g., [1]). These probes generally operate at frequencies above $0.5 \mathrm{GHz}$ (up to 2-3 GHz), i.e., in a frequency range that minimizes the effects associated with the imaginary part of $\varepsilon$. TDR probes are expected to estimate SWC to an accuracy of approximately $2 \%$ without soil specific calibration in coarse-grained soils [1].

In the second group, capacitor sensors use a fixed frequency or frequency shift and operate in the frequency range between approximately 5 and $150 \mathrm{MHz}$. The use of this frequency range leads to two difficulties: (i) the measurement can be affected by dielectric dispersion (i.e., real component $\varepsilon^{\prime}$ is frequency dependent) mainly due to clay minerals (e.g., [2]) and (ii) the measurement cannot fully separate the dielectric response associated $\varepsilon$ ' and the conduction phenomena associated with $\sigma_{D C}$ (see equation (1)). In most cases, a soil-specific or sitespecific calibration is mandatory. However, compared to TDR probes, the geometry of impedance sensors is more adaptable for short electrodes and borehole applications and thus allows interesting methodological developments, as explained below.

Regarding field applications, HF electromagnetic sensors are often considered as "point" measurements due their limited "support" (i.e., the volume in which the average value of SWC is obtained). Ground Penetrating Radar (GPR), a geophysical method, offers potential to overcome this limited spatial resolution. GPR is based on the transmission and reflection of HF (typically between $10 \mathrm{MHz}$ and $1 \mathrm{GHz}$ ) electromagnetic waves within the subsurface. The objective here is to measure following different geometrical arrangements of transmitter and receiver antennas, the velocity of these electromagnetic waves directly linked to the relative permittivity $\varepsilon$. GPR like numerous other geophysical techniques is a highresolution method (down to a few $\mathrm{cm}$ or $\mathrm{dm}$ ) able to quantify the spatial variations of relative permittivity $\varepsilon$ over large areas (easily several hundreds of $\mathrm{m}^{2}$ ). It is often argued that GPR is an important technique to reduce the gap between "point" measurements and regional approaches, such as remote sensing techniques.

\subsection{Recent methodological advances}

\subsubsection{Wireless sensing applications}

Major technical progress regarding HF electromagnetic sensors will likely be achieved with the use of capacitance sensors and more precisely the family of the TDT sensors. These low-cost sensors that use the oscillation frequency of a ring oscillator to estimate $\varepsilon$, currently show interesting features suitable for wireless sensing applications. All electronics are included in the head of the probe removing the need for long cables and multiplexers (e.g., [3]). This advance has opened the opportunity to develop "smart" SWC monitoring systems in the field.

However, one has to keep in mind that wireless or distributed sensing applications still suffer from the following disadvantages:

(a) These methodologies are not standardized; i.e., several commercial probes with different sizes and operating frequencies exist.

(b) Consequently, sensor calibration is often required and may be fastidious in the field when different types of soils are present.

(c) A SWC measurement is often a "point" measurement at the field scale and the interpolation of data between sensors still remains a geostatistical challenge.

\subsubsection{Multi-functional sensing probes}

The integration of various types of data in a single sensor is particularly attractive because it (a) minimizes the soil disturbance and (b) validates deterministic modeling tools that couple water flow, solute transport, heat fluxes and from geotechnical perspective, hydro-mechanical processes. Recent works on this topic include the following developments:

- A combined tensiometer-moisture probe: a coiled TDR device that can be wound around a high suction tensiometer [4] (Figure 1). The complex geometry of this TDR probe requires a soilspecific calibration. The main objective here is to obtain the soil water retention curve in situ by measuring both SWC and suction.

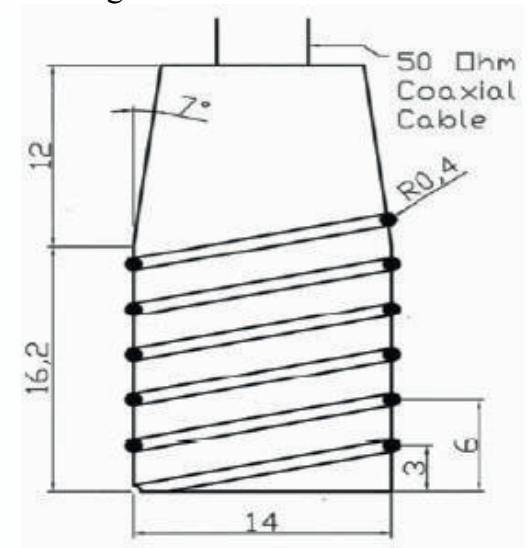

Figure 1. Schematic of the tensiometer housing and coiled TDR probe (dimensions in $\mathrm{mm}$ ) (From [4]).

- A thermo-TDR probe which combines dualprobe heat pulse (DPHP) with TDR technology (e.g., [5]). This probe estimates both thermal properties and electromagnetic properties (relative permittivity and DC conductivity). 
- Combined penetrometer-moisture probes. These tools address the study of soil compaction and the investigation of the heterogeneous hydrological properties of a foot-slope area (e.g., [6]).

\subsubsection{Multi-frequency and high-resolution GPR}

During the past decade, several advances in the GPR systems have been made. Increases in data-acquisition speeds have made it possible to collect more data in a short time whereas new global positioning systems (GPS) allow higher resolution information for the spatial registration of data. Moreover, multi-channel/multifrequency systems now provide varying investigation depths and vertical resolving capabilities.

All these developments have stimulated researches to improve in situ SWC monitoring especially through timelapse or 4D acquisitions. Recent studies (e.g., [7-8]) have shown the abilities of these new techniques to monitor dynamic hydrological processes and specific transient spatial changes in SWC in simple geological contexts (i.e., sandy formations).

However, validation of these techniques in more complex geological contexts still remains. Indeed, a radar image or a radargram acquired in a natural system results from different heterogeneities whose origins are not necessarily hydrological. Moreover, the recent work of [9] performed in more complex geological contexts suggests that the highest frequencies $(900 \mathrm{MHz}$ in their case) are preferred to optimally characterize soil moisture conditions.

\section{Low-frequency electromagnetic methods}

\subsection{Physical principles and methodologies}

Among low-frequency (LF) electromagnetic methods, the direct-current (DC) resistivity method is likely the most known in geotechnical engineering. From a practical point of view, a DC current (in fact a low-frequency alternating current) is injected into the soil by a pair of electrodes and the value of soil electrical resistance is obtained by measuring the resulting difference in voltage on two other electrodes. The soil resistivity is then calculated from the measured resistance by multiplying the latter by a "geometrical factor" that depends on the arrangements of different electrodes and the geometry of the soil sample in a laboratory experiment.

Soil resistivity is primarily sensitive to the number of electrical charge carriers (ions) in the interstitial solution and thus to the solution content. When smectite-type clay minerals are present, a significant part of these charge carriers are captured in the so-called electrical double layer at the clay minerals surface and contribute to an electrical surface conduction which has to be considered in the interpretation of soil electrical data.

The most popular geometrical configuration in the field is currently 2D electrical resistivity tomography (ERT) (also recently called electrical resistivity imaging,
ERI). The main objective here is to obtain a 2D distribution of electrical resistivity in the subsurface. For this purpose, a multi-electrode system consisting of a series of electrodes attached to a resistivity meter is used. A current is injected by two source electrodes and the resultant potential distribution is measured at two or more receiving pairs of electrodes. In the ERT method, this procedure is repeated for as many combination of source and receiver electrodes as desired. Usually this procedure may involve the collection of several hundred (or even more) multi-electrode combinations. This collection of apparent resistivity values is then inverted by appropriate (commercial) inversion software to obtain a resistivity image or an interpreted 2D resistivity section.

It is worth noting that recent case studies have illustrated the ability of 3D ERT methods to monitor soil moisture dynamics in the field (e.g., [10-11]) and in laboratory (e.g.,[12]): this 4D ERT methodology is often referred to a time-lapse ERT method.

\subsection{Recent methodological advances}

\subsubsection{Multi-functional devices}

In the laboratory, a new electrical probe has been designed to monitor SWC changes in a triaxial apparatus [13]. This probe is composed of four circular electrodes (1.5 $\mathrm{mm}$ diameters) disposed in a square-grid scheme (inter-electrode distances of $6 \mathrm{~mm}$ ) as presented in Figure 2. A dielectric matrix (Araldite epoxy resin) is used to accommodate the electrodes in order to avoid any electrical current lines between them.

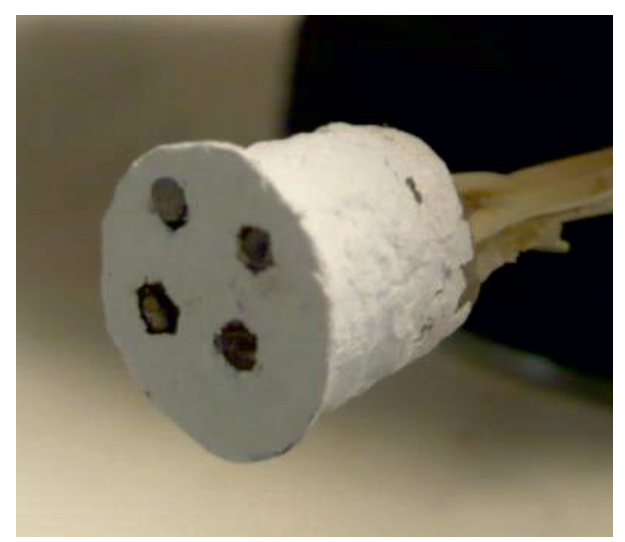

Figure 2. In-house constructed resistivity probe (the four electrodes are separated by distances of $6 \mathrm{~mm}$ ) (from [13]).

This electrical probe is associated with a specific holding system to ensure a good electrical contact with the triaxial specimen. In the triaxial experiments performed by [13], a suction probe is also fixed on the triaxial sample opposite to the electrical probe.

In the same way, to obtain a multi-functional device, a modified oedometer cell developed by [14] simultaneously measures the electrical resistivity and the settlement of the sample. The oedometer ring, which is composed of Teflon (an electrical insulating material) allows for the coupling of a classical oedometer procedure and an electrical resistivity measurement. The 
two metallic porous plates of the oedometer cell are used as the current and potential electrodes. Although this dual-function device was initially designed to study the relationship between soil resistivity and soil compaction, it can be easily used to monitor SWC of a soil sample if a proper calibration of the measured resistivity is conducted.

\subsubsection{Electrical mapping methods}

\section{Automatic resistivity profiling (ARP)}

To our knowledge, the most rapid geophysical method to map electrical resistivity over large areas is the automatic resistivity profiling (ARP, formerly, multi continuous electrical profiling). The ARP method is currently used in archeology and soil science to map electrical resistivity at different depths of investigation and over large areas (typically a few ha). However, surprisingly, this technique is not so commonly used in civil engineering although some case studies illustrating its capacity to map SWC do exist (e.g., [15-16]).

In practice, ARP is a mobile multi-electrode system pulled by an off-road vehicule and coupled to a GPS device. The example given in Figure 3 is a $\mathrm{V}$-shaped array composed of three receiving/voltage pairs of electrodes (three "dipoles" $\mathrm{M}_{1} \mathrm{~N}_{1}, \mathrm{M}_{2} \mathrm{~N}_{2}$ and $\mathrm{M}_{3} \mathrm{~N}_{3}$ ) and one injection pair of electrodes $(\mathrm{AB})$ inserted into the topsoil. The distance between the injection electrodes and the voltage measurement electrodes is $0.5 \mathrm{~m}$ for the $\mathrm{M}_{1} \mathrm{~N}_{1}$ dipole, $1 \mathrm{~m}$ for the $\mathrm{M}_{2} \mathrm{~N}_{2}$ dipole and $2 \mathrm{~m}$ for the $\mathrm{M}_{3} \mathrm{~N}_{3}$ dipole, which correspond approximately to three different depths of investigation (Figure 3b).

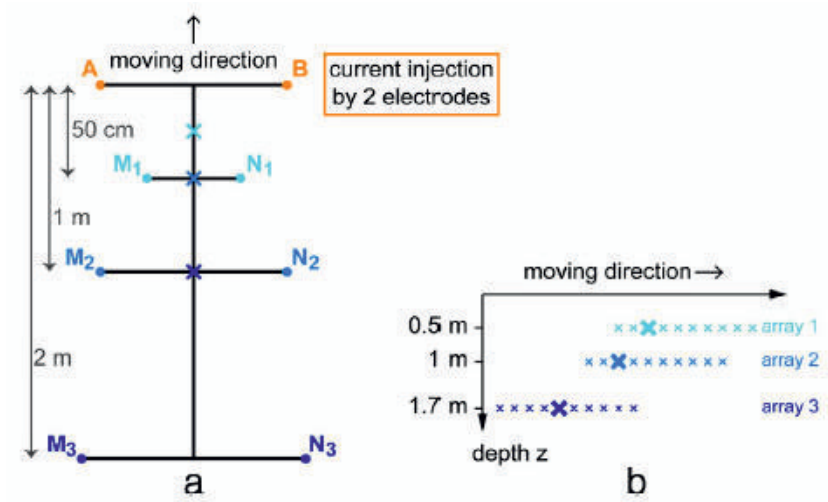

Figure 3. a) Configuration of an ARP device (crosses show the three measurement locations) and b) principle of multi-depth measurements (crosses show the depths of investigation at 0.5 , 1 and $1.7 \mathrm{~m}$ of the three measurements corresponding to the three electrode arrays) (from [17]).

\section{Capacitive resistivity mapping}

The conventional electrical resistivity method has a major disadvantage; direct (galvanic) contact of electrodes with soil is required. This requirement can be problematic in areas with very rigid and/or impenetrable ground cover (i.e., dry and cracked soils, highways, hard rocks and permafrost). Moreover, galvanic measurements on a high resistive surface induce high contact impedances that decrease the accuracy of resistivity measurements. In this context, electrostatic method, often referred to as a capacitive resistivity method is an emerging geophysical technique with the potential to overcome both problems. Indeed following this method, no physical contact between the instrument and the surface of the measured material is required; two poles located in the air, outside the medium under study, produce the electrical field that generates electrical conduction in soil. These transmitter poles constitute an open capacitor whose complex impedance can be calculated from the voltage measured between two (or more) receiver poles.

The recent work of [18] has shown the capability of the electrostatic method to capture the water movement in the unsaturated zone. Moreover, it is worth noting that the electrostatic method can be used in an ARP configuration [19] (Figure 4).

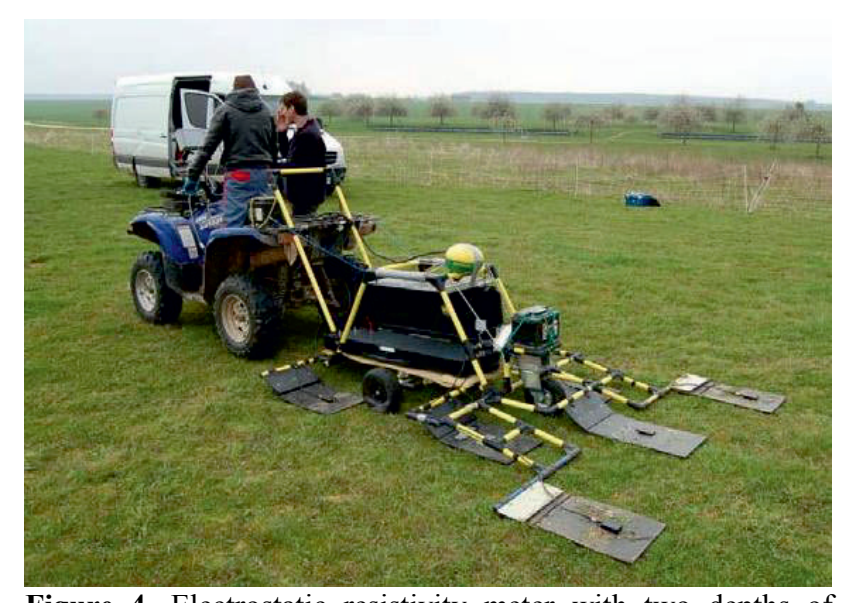

Figure 4. Electrostatic resistivity meter with two depths of investigation for soil mapping at large scale (from [19]).

\subsubsection{Spectral induced polarization method}

When the soil resistivity measurement is repeated for different frequencies of the injected current (typically in the range of $0.1 \mathrm{~Hz}$ to $1 \mathrm{kHz}$ ), a spectrum of soil resistivity can be obtained: this methodology refers to the spectral induced polarization (SIP) method or impedance spectroscopy in laboratory applications. In practice, a spectrum of soil complex impedance (or complex resistivity) is measured because a phase shift exists between the injected electric current and measured voltage. The spectrum of this phase shift demonstrates the presence of polarization mechanisms occurring at low frequencies (typically $<1 \mathrm{kHz}$ ) which are closely related to the pore-scale properties of soil that in part control hydraulic permeability (e.g.,[20]). Moreover, as for conventional electrical resistivity method, the spectrum of the in-phase (or real) component of complex impedance is directly associated with electrical conduction phenomena which in turn are related to the solution/water content.

Finally, this means that SIP spectra allow to estimate (a) SWC but also (b) some microstructural features (e.g., distribution of pore sizes or grain sizes, see Figure 5) which can be related to water permeability or specific surface.

In practice, the main difficulties with obtaining accurate SIP measurements are due to (a) the effects of 
electromagnetic and/or capacitive coupling between the wiring that is used to connect the electronics to electrodes and (b) electrode polarization effect (non-polarizing electrodes have to be specifically designed). Moreover, despite recent theoretical advances, the physical meaning of SIP spectra acquired on a broad frequency bandwidth is still an intensive area of research (e.g.,[21]).

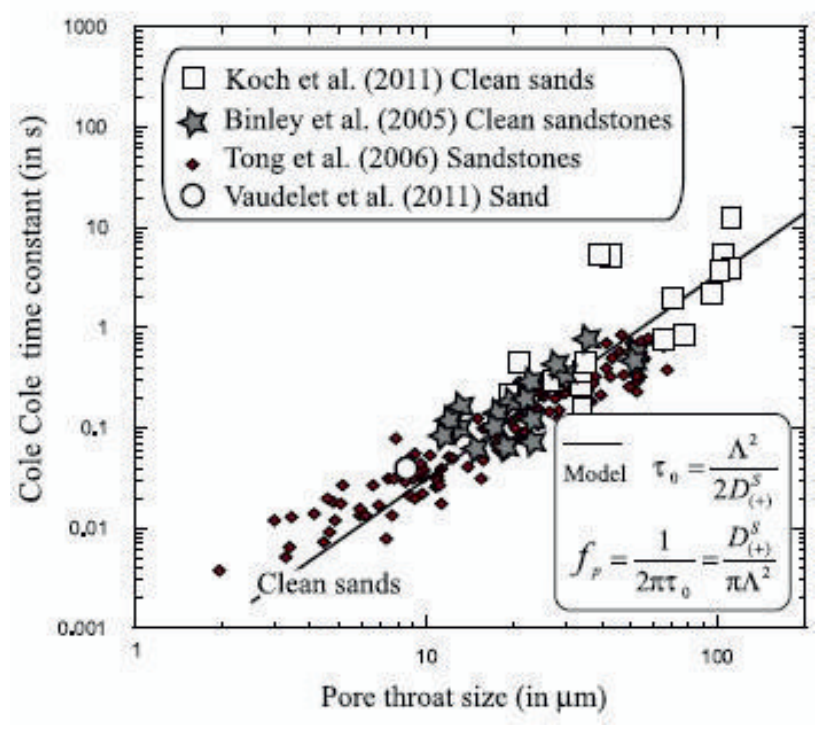

Figure 5. Pore-throat size versus Cole-Cole relaxation time inferred from SIP data obtained from clean sands (from [20]).

\section{Emerging and promising methods}

\subsection{Nuclear magnetic resonance techniques}

Nuclear magnetic resonance (NMR) methodology was originally developed in the oil industry. As SIP method, it deserves better recognition in civil engineering because this method is able to determine SWC and may provide some precious microstructural features (e.g., distribution of pore sizes) of geomaterials.

This method is based on the existence of a strong magnetic moment of the proton in the hydrogen nucleus. At thermal equilibrium in a static magnetic field, the volume of interstitial water in a soil sample exhibits a small net magnetic moment that results from the sum of all the magnetic moment associated with each of the protons in the volume. This net magnetic moment is aligned with the static magnetic field (usually Earth's magnetic field in the field) and its modulus, called $E_{0}$, is proportional to the number of protons. If the number of protons of the hydrogen nuclei is assumed to be proportional to volumetric SWC, it implies that the modulus $E_{0}$ is also proportional to volumetric SWC, which can be then estimated by an ad hoc calibration.

In fact, in a classical NMR relaxometry experiment, the moments of protons, initially at thermal equilibrium are perturbed by an energizing pulse tuned to the Larmor frequency which is an intrinsic physical property of a given nucleus. If this pulse is applied and then removed, these moments precess from their thermal equilibrium and then relax back to this same thermal equilibrium. As these moments relax, they emit a measurable magnetic signal which allows for the calculation of two parameters: relaxation times $T_{1}$ and $T_{2}$ associated with relaxation longitudinal and transversal to the static field, respectively. In the context of geomaterial, the $\mathrm{T}_{2}$ distribution (with respect to the proportion of the magnetic field relaxing in the transverse direction) is often related to the pore size distribution (e.g., [22]). This explains the empirical relationships between NMR $\mathrm{T}_{2}$ and hydraulic permeability existing in the petroleum literature. However, it should be noted that the use of NMR is problematic in presence of (a) iron metals associated with large magnetic susceptibilities (ferromagnetic materials) and (b) clay minerals associated with very short $\mathrm{T}_{2}$.

Regarding laboratory and ground-based NMR methods, recent advances mainly address the following issues:

(a) Understanding the link between NMR measurements and hydraulic parameters of the unsaturated zone i.e., the soil water retention curve. With respect to this objective, recent laboratory investigations suggest that quantitative linear relationships exist between some geometrical features of the $\mathrm{T}_{2}$ distribution (peak value and area) and the degree of saturation of silts [23]. This observation needs verification for others soils.

(b) Improvement of the in situ technique and inversion procedure to image SWC from field data. With regard to this issue, 3D numerical tools validated by field measurements are now available (e.g., [24]).

\subsection{Methods based on fiber optic temperature sensing systems}

Among the numerous optical methods used to determine SWC, those based on distributed temperature sensing (DTS) systems using fiber optic cables are growing in surface water hydrology $[1,25]$. Their main advantages lie in their high spatial and temporal resolution in comparison with "point" measurements associated with HF electromagnetic sensors. Recent works suggest that DTS technology could provide estimates of SWC of lengths up to $10 \mathrm{~km}$ with spatial resolution on the order of $1 \mathrm{~cm}$ and sub-daily temporal resolution [26]. In these DTS methods, temperature is considered as a tracer of water movement; the temperature measurement is based on the thermal sensitivity of the relative intensities of backscattered Raman Stokes and anti-Stokes that arise from collisions with electrons in the core of the glass fiber [27].

From a practical point of view, the DTS methods to estimate SWC can be divided in two groups. In the first group, the passive methods simply record temperature variations of soil induced by the diurnal temperature cycle; no external source of energy is required. Its application may be questionable when the thermal response to the amplitude of diurnal cycle is not large enough to allow accurate estimation of SWC. The second group of methods refers to the active methods that couple a DTS system and heat source (or with a heat pulse method)

Recent advances regarding DTS systems mainly concern the active methods for which the works focused 
on the performances of heated optic cables (accuracy and suitability), the technology required to their insertion into the ground [27-28] and the limitations for long-term field applications [26].

\section{Summary and concluding remarks}

To summarize, our review highlights the following current trends and areas of research for indirect determination of SWC:

- Integration of different types of sensors to design new multi-functional devices. These multifunctional devices allow for experimentally addressing the numerous coupled hydro-mechanical problems existing in geomechanics.

- Miniaturization of low-cost and easily installed electromagnetic sensors. Recent advances in electronics allow to address the emerging smart SWC monitoring in the field (e.g., wireless sensing applications).

- Data fusion and assimilation. This aspect is a consequence of the previous points. Combining different and numerous data from multiple modalities with different sampling rates and spatial resolutions is becoming an emerging domain of research.

- Physical understanding of spectra or distributions measured in SIP and NMR methods. The practical objective here is to obtain by a single set of data, both SWC and microstructural features of the studied geomaterial.

- Improvements of inversion techniques. Improvements in geophysical inversion techniques combined with fast data acquisitions (thanks to technological advances as well) allows time-lapse and 4D methodologies to monitor SWC both in the laboratory and in the field.

However, it should be emphasized that accuracy of most indirect methods described here is still problematic in saline soils and clay geomaterials for which a materialspecific calibration is always required. Regarding the latter (clay soils and clay rocks), the understanding and modeling of their physical parameters with respect to their hydration state remains a challenging area of research.

\section{References}

1. Robinson D. A., Campbell C. S., Hopmans J. W., Hornbuckle B. K., Jones, S. B., Knight R., Wendroth O. Vadose Zone J., 7, 1 (2008)

2. Cosenza P., Tabbagh A. Appl. Clay Sci., 26, 1 (2004).

3. Qu W., Bogena H. R., Huisman J. A., Vereecken H. Vadose Zone J. 12, 2 (2013).

4. Toll D. G., Hassan A. A., King J. M., Asquith J. D. Proc. of the 18th International Conference on Soil Mechanics and Geotechnical Engineering, Paris. France, pp. 1199-1202 (2013).

5. Zhang B., Han C., Yu X. B. J. Rock Mech. and Geotech. Eng., 7, 155-162 (2015).
6. Masaoka N., Kosugi K. I., Yamakawa Y., Mizuyama, T., Tsutsumi, D. Vadose Zone J. 11, 2 (2012).

7. Klenk P., Jaumann S., Roth K.. Hydrol. and Earth Syst. Sci., 19, pp. 1125-1139 (2015).

8. Mangel A. R., Lytle B. A., Moysey S. M. The Leading Edge. 34, pp. 190-196 (2015).

9. Steelman C. M., Endres, A. L. J. Hydrol. 436, pp. 51-66 (2012).

10. Chambers J. E., Gunn D. A., Wilkinson P. B., Meldrum P. I., Haslam E., Holyoake S., Wragg J. Near Surf. Geophys. 12, pp. 61-72 (2014).

11. Gance J., Malet J. P., Supper R., Sailhac P., Ottowitz, D., Jochum B. J. of Appl. Geophys. in press (2016).

12. Clement R., Moreau S. J. Appl. Geophys. In press (2016).

13. Munoz-Castelblanco JA, Delage, P., Pereira, JM, Cui, YJ. Géotechnique. 62, pp. 595-604 (2012).

14. Ghorbani, A., Cosenza P., Badrzadeh Y., Ansari A. Eur. J. Environ. Civil Eng. 17, pp. 84-98 (2013).

15. Besson A., Cousin I., Bourennane H., Nicoullaud B., Pasquier C., Richard G., King, D. Eur. J. of Soil Sci. 61, pp. 120-132 (2010).

16. Bourennane H., Nicoullaud B., Couturier A., Pasquier C., Mary B., King D. Geoderma. 183, pp. 32-40 (2012).

17. Buvat S., Thiesson J., Michelin J., Nicoullaud B., Bourennane H., Coquet Y., Tabbagh A. Geoderma. 232, 317-327 (2014).

18. Niu Q., Wang Y. H., Zhao K. J. of Appl. Geophys. 106, 119-127 (2014) .

19. Dabas M., Tabbagh A., Flageul S. 3rd Global Workshop on Proximal Soil Sensing, Potsdam, Germany. pp 27 (2013).

20. Revil A., Florsch N., Camerlynck C. Geophys. J. Intern. 198, pp. 1016-1033 (2014).

21. Okay G., Leroy P., Ghorbani A., Cosenza P., Camerlynck C., Cabrera J., Florsch N, Revil A. Geophys. 79, pp. E353-E375 (2014).

22. Tian H., Wei C., Wei H., Yan R., Chen P. Appl. Mag. Reson. 45, pp. 49-61 (2014).

23. Chen P., Wei C. F., Liu, J., Xue, W. J. Unsaturated Soil Mechanics-from Theory to Practice: Proceedings of the 6th Asia Pacific Conference on Unsaturated Soils, Guilin, China. pp 181. CRC Press, (2015).

24. Jiang C., Müller-Petke M., Lin J., Yaramanci U. J. Appl. Geophys. 116, pp.17-27 (2015).

25. Bakker M., Caljé R., Schaars F., van der Made K. J., de Haas S. Water Resour. Res. 51, pp. 2760-2772 (2015).

26. Sourbeer, J. J., Loheide, S. P. Hydrol. Process. In press (2015).

27. Tyler S. W., Selker J. S., Hausner M. B., Hatch C. E., Torgersen T., Thodal C. E., Schladow S. Water Resour. Res. 45, 4 (2009).

28. Coleman, T. I., Parker, B. L., Maldaner, C. H., \& Mondanos, M. J. J. Hydrol. 528, pp. 449-462 (2015). 